\title{
Fatigue under Complex Loading History in a Random Vibration Environment - a Survey
}

\author{
Yi Gong and Michael P. Norton \\ Department of Mechanical and Materials Engineering, University of Western Australia, Nedlands, WA 6907, Australia
}

In this paper, various fatigue life regimes, life assessment approaches and their implications in design are summarised. Emphasis is placed on modelling fatigue under complex loading history in random vibration environments. Factors affecting the fatigue life of materials and structural components are presented. Multi-axial random fatigue problems are also discussed.

\section{INTRODUCTION}

Fatigue of materials and structures can be defined as "the progressive localised permanent structural change occurring in a material subjected to conditions which produce fluctuating stresses and strains at some point or points and which may culminate in cracks or complete fracture after a sufficient number of fluctuations". ${ }^{1}$ Most of the engineering structures, such as aircraft, bridges, offshore platforms, pipelines, pressure vessels, etc., operate under repeated thermal and/or mechanical vibrational loading conditions. Cyclic stresses and strains are thus induced which makes fatigue failure one of the most frequent and primary failure modes in engineering applications. It is estimated that between 50 percent and 90 percent of all mechanical failures in metallic structures are fatigue related. ${ }^{2}$ Therefore, fatigue failure prevention during service period and fatigue life assessment in the design stage have long been a major concern in operation process and engineering design, especially for rotating machinery and aircraft. Although the earliest research on fatigue problems can be traced back to the first half of the nineteenth century, ${ }^{3}$ the fatigue properties of a wide variety of materials, metallic and non-metallic, brittle and ductile, monolithic and composite, remain an active research topic. It is not until recently that the basic rule of design taking into account the possibility of fatigue failure and its prevention was established based on the Palgmren-Miner linear cumulative damage rule. One of the possible reasons for this is that fatigue is a highly complicated phenomenon when practical service conditions, such as loading, environment and manufacturing process are concerned.

Special difficulties arise when the inherent random properties of fatigue are considered. It has been observed well that even under carefully controlled conditions large scatters of fatigue damage exist. It is generally believed that this uncertainty comes from every aspect relating to the component of the specific material under service, for instance, the microstructural properties of the material, the loading properties, and environmental conditions, etc. Consequently, the stochastic modelling of fatigue problems by adopting probability and statistics theory seems to be inevitable for a quantitative description of the fatigue process. Basically, stochastic modelling of fatigue problems can be one of the following or their combination: the modelling of the random loading, the modelling of the fatigue damage process, and the modelling of the random scatter of fatigue life. For the purposes of this review, however, fatigue problems involving random loading will be discussed.

From an engineering standpoint, the total fatigue life of a structural component has two phases, namely, the crack initiation phase, which is from the application of the first load cycle to the presence of a technically detectable crack, and the crack propagation phase, which starts with the end of the crack initiation phase and ends when the crack reaches a predetermined critical size or when failure occurs.

Bearing this in mind, there are consequently two major approaches associated with the evaluation of fatigue life:

- The phenomenological approach (total life approach), which characterises the total fatigue life as a function of stress or strain level by embodying the crack nucleation, initiation and propagation stages into a single, experimentally characterizable formulation. Here the fatigue life of a component is defined as the total number of cycles from the initially uncracked state to final failure. This is the basis of the Safe-life design philosophy where crack initiation is of major concern.

- The defect-tolerant approach (fracture mechanics approach), which mainly considers the crack growth rate under fatigue loading of an initially flawed component. In this case the fatigue life is defined as the number of cycles from an initial crack length to a predetermined critical length or to final failure. This constitutes the basis for the Fail-safe design method where the existence of cracks is allowed by redundant structural components.

Limited in its ability to have a direct insight into the fatigue damage process itself, the phenomenological approach still finds wide application in engineering practice due possibly to the availability of large amount of experimental data and the simplicity of application. Another possible reason lies in the fact that it is extremely difficult in practice to make a clear demarcation between the crack initiation stage and the crack propagation stage. On the other hand, considerable care must be exercised when applying the fracture mechanics approach since, although it is a useful method for fatigue crack growth analysis, the prediction will be only as good as the assumptions made, such as an existing crack, the size of the initial crack, the shape of the crack and the geometrical configuration of the structures concerned. ${ }^{4}$

This review is aimed at:

- Summarising various fatigue life regimes and their implications; 